\title{
Calcifying Epithelial Odontogenic Tumor of The Anterior Maxilla: An Unusual Location
}

\author{
Zeeba Shamim Jairajpuri, Mohd Jaseem Hassan*, Manal Ashraf Ali and Sujata Jetley
}

Department of Pathology, Hamdard Institute of Medical Sciences and Research (HIMSR), Jamia Hamdard, New Delhi, India.

\begin{abstract}
Calcifying epithelial odontogenic tumor (CEOT) is a benign tumor of odontogenic epithelium.It is a locally invasive neoplasm with a characteristic feature of having amyloid.The mandible is twice more commonly affected than the maxilla and in the maxilla, posterior location is the preferred site of involvement. Here we present a case in a 50 years old male patient who presented with a non-tender hard swelling of the anterior maxilla measuring $2.5 \times 1.5 \mathrm{~cm}$, just right to the midline and at the base of the lateral incisor and canine. Imaging revealed a radiolucent lesion in the maxilla with small foci of calcification without any evidence of impacted tooth. Thus a provisional diagnosis of an Ameloblastoma was made. A local excision of the swelling was done alongwith extraction of the lateral incisor and canine. On histopathological examination, a diagnosis of Calcifying epithelial odontogenic tumor was rendered.
\end{abstract}

Keywords: Key Words: Benign Tumor Jaw, CEOT, Maxilla, Pindborg

\section{Introduction}

Calcifying epithelial odontogenic tumor (CEOT) is a benign tumor of odontogenic epithelium,first described by the Danish pathologist, Dr. Jens J. Pindborg in 1955. ${ }^{1}$ CEOT is listed as a benign tumor of odontogenic epithelium with mature fibrous stroma without odontogenic mesenchymeby World Health Organization. ${ }^{2}$ It is a "locally invasive neoplasm with a characteristic feature of having amyloid which tends to calcify". ${ }^{2}$ The origin of CEOT is said to arise from the enamel organ's stratum intermedium in the tooth development stage, a few also believe that they may arise from the remnants of the dental lamina of initial stages of odontogenesis. ${ }^{3}$

\section{Case Report:}

A 50 year old male presented with swelling in the right maxilla of 3 months duration. There was no associated pain or nasal stuffiness. Extra-oral examination showed a mild swelling along the right naso-labial fold. The overlying skin was normal. An intra-oral examination revealed a firm to hard swelling of the anterior maxilla, just right to the midline and at the base of the lateral incisor and canine. The swelling measured about $2.5 \times 1.5 \mathrm{~cm}$ and was non-tender. The overlying mucosa and the adjacent teeth were unremarkable. The patient did not give any previous or recent history of tooth extraction or any other dental complaints or procedures.

Imaging revealed a radiolucent lesion in the maxilla with small foci of calcification. There was no evidence of any impacted tooth. A provisional diagnosis of an ameloblastoma was considered. A local excision of the swelling was done alongwith extraction of the lateral incisor and canine, and the specimen sent for histopathological examination.

Histopathology, revealed an unencapsulated tumor composed of sheets and islands of polyhedral epithelial cells with moderate to abundant eosinophilic cytoplasm and distinct cell borders. The cells have pleomorphic nuclei,but no mitosis. The surrounding stroma is fibrous to hyalinized. There are numerous amorphous pale eosinophilic rounded masses of amyloid like material, with few showing calcification, and surrounding acute on chronic inflammatory infiltrate (Fig-1). Thus the tumor was diagnosed as CEOT of right anterior maxilla.

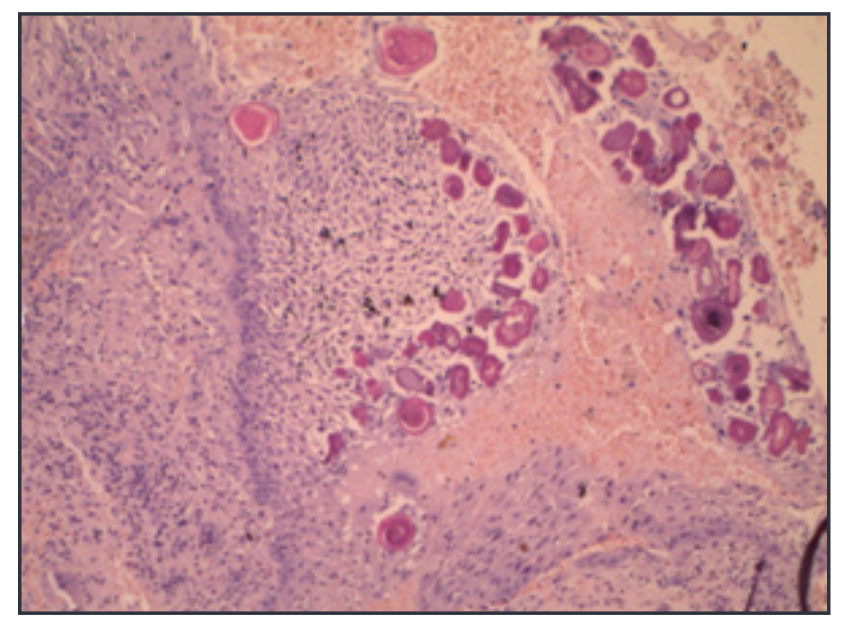

Fig.1: Microphotograph showing sheets and islands of polyhedral epithelial cells in a fibrous to hyalinized stroma with presence of numerous amorphous pale eosinophilic rounded masses of amyloid like material, with few showing calcification.(H\&Ex100) 


\section{Discussion}

The mean age of presentation is around 40 years of age, with arange of 20 to 60 years without any sex predilection, our patient was 50yrs old. ${ }^{4}$ Younger patients have also been reported, recently CEOT has been described in two female children of the ages of $12 \& 13$ years respectively. ${ }^{5}$ Majority of the cases $(95 \%)$ are intraosseous, as in the present case, ${ }^{6}$ however a small section of them happen to be extraosseous (gingival). The mandible is known to be twice more commonly affected than the maxilla, with a predilection for the ramus. ${ }^{7}$ In cases affecting the maxilla, the posterior locationis more common with the premolar to molar ratio being $1: 3{ }^{8}$ The present tumor was located in the anterior maxilla at the base of the lateral incisor and canine, which is an uncommon presentation. About $50 \%$ of the tumors are associated with an un-erupted tooth, most commonly the third molar. ${ }^{2}$

Clinical presentation of CEOT varies, from being asymptomatic, accidently discovered on radiology, to being symptomatic enough for the patient to seek medical help. The patient may have nasal stuffiness and pain, besides the swelling which causes cosmetic problems. Also due to tooth resorption in advanced cases, there may be mobility of involved tooth or tooth tipping. ${ }^{9}$

Histopathologically, CEOT shows a population of polyhedral epithelial cells with distinct borders and intercellular bridges. There is anisonucleosis with pleomorphic nuclei. The surrounding stroma is fibrous to hyalinized with amyloid deposits and concentric lamellated calcifications, called Liesegang's rings. These rings though not exclusive, are quite characteristic of CEOTs. Tumors without calcification have also been reported, and are said to be poorly differentiated with a high rate of recurrence. ${ }^{10}$ Ahistological subclassification has also been proposed, dividing tumors into four patterns based on cell arrangement, calcifications and the amount of amyloid present. However the authors do not claim any prognostic significance of these subdivisions. ${ }^{11}$

Many variants of CEOT have been reported, namely; clear cell,non calcifying CEOT with Langerhans cells, CEOT with cementum and bone, mixed tumor with ameloblastoma and combined adenomatoid odontogenic tumor and CEOT. ${ }^{12}$ Multifocal presentation of CEOT has also been reported, involving both mandible and maxilla in a 50 year male. ${ }^{13}$ Recurrence rates of upto $14 \%$ have been seen, with the clear cell variant being the most notorious. ${ }^{14}$

Malignant transformations in CEOTs have been reported in literature, with threecases showing evidence of metastasis. Malignant transformation is determined by the presence of increased and abnormal mitosis, focal necrosis and an increased proliferative index. Aggressive treatment of maxillary CEOTs is recommended, as they are unencapsulated and grow rapidly. ${ }^{4}$ Primary intraosseous squamous cell carcinoma is a close histopathological differential for CEOT.

\section{Conclusion}

CEOT though rare, are exclusive to the maxillofacial skeleton. The reported case involves the unusual location of the anterior maxilla, as opposed to the more common posterior mandible. A close follow up is advised in all cases, in view of the invasive nature of the tumor.

\section{References}

1. Pindborg JJ. Calcifying epithelial odontogenic tumors. ActaPatholMicrobiol Scand. 1956;71:111.

2. Takata T, Slootweg PJ. Calcifying epithelial Odontogenic tumour. In Barnes L, Eveson JW, Reichart P, Sidransky D. IARC World Health Organisation Classification of Tumours. Pathology and Genetics of Head and Neck Tumours. Lyon:IARC press;2005.pp 302-303.

3. Reichart PA, Philipsen HP, editors. Odontogenic tumors and Allied lesions. London: Quintessence Publishing; 2004. pp. 43-59.

4. Philipsen HP, Reichart PA. Calcifying epithelial odontogenic tumour: biological profile based on 181 cases from the literature. Oral Oncol2000;36:17-26.

5. Mohanty S, Mohanty N, Routray S, Misra SR, Vasudevan V. Calcifying epithelial odontogenic tumor, a rare presentation in children: Two case reports. J Indian SocPedodPrev Dent 2014;32:149-51

6. Ai-Ru L, Zhen L, Jian S. Calcifying epithelial odontogenic tumors: A clinicopathologic study of nine cases. J Oral Pathol 1982;11: 399-406.

7. Houston GD, Fowler CB. Extraosseous calcifying epithelial odontogenic tumor: Report of two cases and review of the literature. Oral Surg Oral Med Oral Pathol Oral RadiolEndod. 1997;83:577-83.

8. Maria A, Sharma Y, Malik M. Calcifying Epithelial Odontogenic Tumour: A Case Report.

9. J Maxillofac Oral Surg. 2010;9:302-306.

10. Singh N, Sahai S, Singh S, SinghS..Calcifying epithelial odontogenic tumor (Pindborg tumor). Natl J Maxillofac Surg. 2011; 2:225-227.

11. Krolls SO, Pindborg JJ. Calcifying epithelial odontogenic tumor. A survey of 23 cases and discussion of histomorphologic variations. Arch Pathol 1974;98:206-10.

12. Ai-Ru L, Zhen L, Jian S. Calcifying epithelial odontogenic tumors: A clinicopathologic study of nine cases. J Oral Pathol 1982;11:399-406. 
13. Chen et al.: A clinicopathologic study on calcifying epithelial odontogenic tumor: with special reference to Langerhans cell variant. Diagnostic Pathology 2014;9:37.

14. Sedghizadeh PP, Wong D, Shuler CF, Linz V, Kalmar JR, Allen CM.Multifocal calcifying epithelial odontogenic
tumor.Oral Surg Oral Med Oral Pathol Oral RadiolEndod 2007; 104:30-34.

15. Franklin $\mathrm{CD}$, Pindborg JJ. The calcifying epithelial odontogenic tumor. A review and analysis of 113 cases. Oral Surg Oral Med Oral Pathol. 1976;42:753-65.

*Corresponding author:

Dr. Mohammad Jaseem Hassan, Department of Pathology, HIMSR, Jamia Hamdard, New Delhi, 110062 India

Email: jaseemamu@gmail.com 